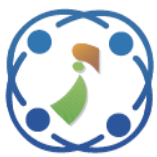

\title{
Robust Video Watermarking using Tchebichef Transform and Singular Value Decomposition on the Selected Frame Based YCbCr Color Space
}

\author{
Andik Setyono ${ }^{1 *}$ \\ De Rosal Ignatius Moses Setiadi ${ }^{1}$ \\ Department of Informatics Engineering, \\ Faculty of Computer Science, Dian Nuswantoro University, Semarang 50131, Indonesia \\ * Corresponding author's Email: andik.setyono@dsn.dinus.ac.id
}

\begin{abstract}
Watermarking is a copyright authentication technique. This research proposes a robust watermarking method with a combination of Tchebichef transformation and singular value decomposition (SVD). To maintain imperceptibility, embedding is done on one of the selected frames. Frames are randomly selected to increase watermark security. Frame selection is based on two integer keys processed by a linear congruential generator (LCG). The selected frame is then converted to its color space from RGB to YCbCr. Y channel (luminance) was selected to be processed by Tchebichef transformation based on block $8 \times 8$, the coefficient 0.0 for each block of the transformed results was selected and collected on a matrix. This matrix is then transformed with SVD and a singular matrix is selected for watermark embedding, this method is done to increase robustness. Based on the test results, the imperceptibility value is very good with an average value of $50.952 \mathrm{~dB}$, based on the PSNR as a measuring tool. Whereas in the robustness aspect, a value of 0.927 is generated based on the results of the measurement of the correlation between the watermark and the original watermark, where these results are the average extraction results without and with various attacks.
\end{abstract}

Keywords: Copyright protection, Linear congruential generator, Singular value decomposition, Tchebichef Transform, Video Watermarking.

\section{Introduction}

Digital data authentication, especially multimedia data, is needed at this time. Internet technology is a necessity especially in the era of the Covid-19 pandemic, which is currently forcing people to reduce activities outside the home. Sharing of data and information, especially multimedia data, is mostly done via the internet. Some digital data spread over the internet has a copyright that must be protected. One of the methods of copyright protection that is widely used is watermarking. Watermarking is a data hiding technique. This method requires cover media which is generally in the form of video, image, or audio [1, 2]. Whereas logos or messages that are embedded as copyright on media covers generally can be in the form of images or text, but in watermarking, copyright in the form of images is preferred because it is more resistant to attack, while copyright in the form of a text can change meaning significantly when there is a change in bit value due to attacks.

One of the requirements for image watermarking is that the copyright size must be smaller than the media cover. Also, the watermark results in musthave aspects such as imperceptibility, robustness, and security [1-4]. Watermarking is quite similar to the steganography method because these two methods are both branches of data hiding science. The difference between watermarking and steganography is the purpose of protection. Watermarking aims to protect the media cover, where the embedded message must be able to survive when manipulation and attacks are carried out on the media cover so that the media cover can still be authenticated as to who owns the copyright $[5,6]$. Whereas steganography prioritizes message security, messages must be guaranteed security, and in the worst conditions, messages should be destroyed than messages read by irresponsible people [6]. 
There are two domains of copyright embedding in data hiding, namely spatial and frequency [7]. The spatial domain has advantages in the imperceptibility and payload aspects of the message, but it is weak against manipulation, where the message will be easily damaged, so this domain is less suitable for the watermarking method. Watermarking research uses more of the frequency domain when embedding copyright because it is more robust against manipulation on media covers, or it can also be done by hybrid domains (frequency and spatial) [8].

Several methods in the frequency domain have been widely applied, i.e. discrete Fourier transform (DFT) [9-11], discrete cosine transform (DCT) [12, 13], discrete wavelet transform (DWT) [14, 15], singular value decomposition (SVD) [16, 17], and discrete Tchebichef transform (DTT) [14, 17, 18]. From these researches, several transformation methods are also combined, this aims to improve the quality of the watermarked image, both in terms of imperceptibility, robustness, and security [19]. In more detail, this research highlights some of the above transformation methods such as SVD and DTT. Various studies using SVD as a transformation, on average are relatively stronger against attack geometry and good stability [20-22]. When SVD is combined with DCT, the watermark is increasingly robust against attacks such as JPEG compression and increased noise [23]. There is another transformation that has many similarities with DCT, namely DTT. DTT has the advantage of simpler computational complexity compared to DCT, even though DTT has identical qualities to DCT [24], so DTT and SVD are combined and proposed in this research.

Currently, research on watermarking is mostly carried out on digital images as cover media. This is because the image has two dimensions that are more complex than the audio, making it more challenging to study. Besides, digital images are relatively more difficult for the human senses to perceive. Watermarking with cover video media is more complex than image and sound media. This is because the video has many frames (images) that are displayed sequentially so that it looks moving. Besides, the video has audio that can be played simultaneously with the frame[5]. Video has the advantage of a larger message payload and very high imperceptibility if the embedded message is very small, for example, it is only embedded in a frame among thousands of frames in the video. Besides, the method of embedding videos can be more diverse, here the watermarking method can be more varied by combining existing methods to increase watermarking security $[5,25]$. But because of its large size, because of the large number of frames and audio data, the process carried out on video watermarking requires relatively longer computation time.

In this research, the watermarking method on the video will be explored further. Several domain transformation methods will be combined with random insertion techniques to increase security. This paper will be divided into five sections, i.e., the first section is an introduction, which discusses the background of researching watermarking videos; the second section is related work, where it is discussed, analyzed and hypotheses are carried out from the researches so that the idea of developing methods emerges; the third section is the proposed method, in the section, it explains the design of the method both with diagrams and narrative steps, and explains the theories used are needed; the fourth section is the presentation of the results and their discussion, and the last section is the conclusions which contain the conclusions and suggestions for the next research.

\section{Related work}

As explained in the introduction, watermarking with video as a media cover is quite challenging and complex. In this section, some related research on video watermarking is explained.

Oliveira et al. [26], proposed a watermarking method based on Discrete Tchebichef Transform (DTT). The motivation for using DTT is because this transformation has low complexity, similar property to DCT, making it suitable for the image and video coding. In his continued research, Oliveira et al. [27], also proves that DTT has very promising results and can be developed to increase resource consumption efficiency with optimal image quality and video coding.

Setyono et al. [17] explicitly implement the DTT method on image watermarking. DTT is combined with SVD transformation based chaos embedding. This method can produce imperceptible watermarks and has dominant resistance to filter media attacks, rescaling, JPEG compression, and salt and pepper when compared to previous methods. This method is also quite good at defending against geometric attacks because of the advantages of the SVD transformation used.

In research conducted by $\mathrm{Li}$ et al. [28], watermarking on video is done using DCT, where the video watermarking is done based on MPEG2 compression. The watermark embedding is done on the DC coefficient based on the $H \& G$ algorithm and quantization to improve performance. A standard video was used in his research. This research produced a watermark with better extraction than the 
standard method and obtained a PSNR value above $37 \mathrm{~dB}$.

Bhardwaj et al. [29] proposed a watermark embedding technique in the video using lifting wavelet transform (LWT). The first time the video is extracted the frames, to select one of the frames for embedding the watermark. The videos used are standard videos such as akiyo, foreman, hall, etc. While the watermark used is a binary image. Embedding is done by doing three levels of LWT, at the first and second level LL sub-band, and at the third level is selected LH sub-band. The LH subband is divided into $4 \times 4$ blocks, then before being embedded, both the watermark and the LH sub-tire blocks are shuffled and then embedded with a quantization process. Next, an inverse shuffle and an LWT inverse were carried out to get a watermarked video. Based on this method, the PSNR value is above $41 \mathrm{~dB}$ for all videos tested.

The watermarking method proposed by Sari et al [23], watermarking is performed on color images, by first converting the RGB color space to $\mathrm{YCbCr}$, then the watermark is embedded in the $\mathrm{Y}$ channel (luminance). This channel contains image intensity, the goal is to increase the robustness of the watermark. Embedding is performed using a combination of DCT and SVD transformations to optimize yield.

Rachmawanto et al. [30], proposed a video steganography technique using LCG-based PVD. LCG is a linear congruential generator, which in this research is used to select a frame to embed a message, quite similar to research [29], where there is a selection of frames before embedding the watermark. The LCG algorithm is quite simple and doesn't take a long time, making it suitable for processing video coding.

From the related work above, it has been explained that several proposed methods can work well in the image. Especially in color images, the conversion of the color space from $\mathrm{RGB}$ to $\mathrm{YCbCr}$ and embedding it on the $\mathrm{Y}$ channel can increase watermark robustness. However, increasing robustness has a risk of reducing imperceptibility. In this research, a video object is used which has more parameters that can be used to get more optimal results, of course, if the appropriate composition is obtained. Videos have many frames, embedding a watermark based on the frame selection process is indeed proven to produce good imperceptibility values, and of course, it can increase watermark security. Besides, the Tchebichef Transformation excels in computation speed because of its low complexity, but it also has properties similar to that of DCT which are strong against attacks. Meanwhile, SVD excels in resistance to geometric attacks. Based on some of the related work that has been proposed, this research proposes a combination of Tchebichef and SVD transformations in video watermarking where the embedding is carried out on one of the selected frames using an LCG generator. The frame color space will be changed from RGB to $\mathrm{YCbCr}$ to improve watermark robustness.

\section{Proposed method}

This section describes in more detail the process of embedding and extracting watermarks on videos using a combination of Tchebichef and SVD transformations.

\subsection{Embedding scheme}

The proposed embedding scheme requires three inputs, namely the original cover video, watermark/copyright, and LCG key. To see the workflow of the proposed embedding method, see Fig. 1. Based on Fig. 1, in detail the process is described as follows:

1. The original cover video is read for the first time, where the cover video data will be used in the embedding process, some of which is the number of frames $(n f)$ and video dimensions.

2. Furthermore, two input variables are needed, as the LCG key, namely variables $a$ and $b$. The LCG requires a variable $m$, but the variable automatic generates based on the number of frames $(m=n f)$. By default, the LCG can be calculated by Eq. (1)[30], because LCG can generate pseudo-random numbers, but because only the first random number is needed, it is based on Eq. (1), a slight modification is done, where the value of $n$ will be limited to 2 , where the initial $n$ value $=1$ so that the values of $Z_{1}$ and $Z_{2}$ are generated. The $Z_{1}$ and $Z_{2}$ values are then added and divided in half and the results are floored to get the frame value that will be embedded with the watermark.

$$
Z_{n}=\left(a \cdot Z_{n-1}+b\right) \bmod m
$$

3. Nilai LCG yang dihasilkan selanjutnya digunakan untuk mendapatkan frame ke-Z yang saat melakukan ekstraksi frame.

4. Setelah didapatkan frame ke-Z, dilakukan konversi ruang warna pada Frame, dari ruang warna RGB menjadi YCbCr dengan Eq. (2) 


$$
\begin{gathered}
{\left[\begin{array}{l}
Y \\
C b \\
C r
\end{array}\right]=\left[\begin{array}{ccc}
0.299 & 0.587 & 0.114 \\
-0.1687 & -0.3313 & 0.5 \\
0.5 & -0.4187 & -0.0813
\end{array}\right]} \\
{\left[\begin{array}{l}
R \\
G \\
B
\end{array}\right]+\left[\begin{array}{c}
0 \\
128 \\
128
\end{array}\right]}
\end{gathered}
$$

5. Next, select the Luminance (Y) channel to perform the Tchebichef transformation by dividing the $\mathrm{Y}$ channel into non-overlapping blocks $(S)$ with a size of $8 \times 8$ pixels, the Tchebichef transformation is carried out using Eq. (3). Y channel chose because this channel represents intensity which contains the core meaning of the image [31].

$$
T_{x y}=\sum_{x=0}^{7} \sum_{y=0}^{7} t_{x}(i) t_{y}(j) S(i, j)
$$

Based on the Tchebichef transformation on Eq. (3) there are $t_{x}(i)$ and $t_{y}(j)$, which are Tchebichef's orthonormal polynomials. Where the degrees $\mathrm{x}$ and $\mathrm{i}$ refer to the abscissa of the $\mathrm{Y}$ channel frame pixels, while $y$ and $j$ are the ordinates of the $Y$ channel frame pixels. $T$ is the result of Tchebichef's transformation based on the pixel spatial value $(S)$ of the Y channel frame. $t_{0}(i)$ can be calculated by Eq. (4), $t_{1}(i)$ can be calculated by Eq. (5), while for $t_{2}(i)$, up to $t_{7}(i)$ can be calculated by Eq. (6).

$$
\begin{gathered}
t_{0}(i)=\frac{1}{\sqrt{8}} \\
\left.t_{1}(i)=2 i+1-8\right) \sqrt{\frac{3}{8\left(8^{2}-1\right)}} \\
t_{x}(i)=\left(\alpha_{1} i+\left(\alpha_{2}\right) t_{u-1}(i)+\alpha_{3} t_{x-2}(i)\right.
\end{gathered}
$$

In Eq (6), there is $\alpha_{1}$ which can be calculated by Eq. (7), $\alpha_{2}$ which is calculated by Eq. (8) and $\alpha_{3}$ calculated by Eq. (9).

$$
\begin{aligned}
& \alpha_{1}=\frac{2}{x} \sqrt{\frac{4 x^{2}-1}{8^{2}-x^{2}}} \\
& \alpha_{2}=\frac{1-8}{x} \sqrt{\frac{4 x^{2}-1}{8^{2}-x^{2}}} \\
& \alpha_{3}=\frac{x-1}{x} \sqrt{\frac{2 x+1}{2 x-3}} \sqrt{\frac{8^{2}-(8-1)^{2}}{8^{2}-x^{2}}}
\end{aligned}
$$

6. Collect each value from $\mathrm{T}(0,0)$ in each block, then collect it on a matrix $\left(M_{d c}\right)$. The value at $\mathrm{T}(0,0)$, was chosen because this value contains the core value of the frame, it is identical to the DC value in DCT [7].

7. Perform SVD transformation on $M_{d c}$, then take singular matrix. Transformasi SVD dapat dilakukan dengan Eq. (10).

$$
S V D=H S V^{T}
$$

8. Where $H$ is the horizontal matrix, $S$ is the diagonal matrix and $V$ is the vertical matrix.

9. On the other hand, read the watermark image, then transform the watermark image SVD, then take the singular matrix.

10. Embed a watermark $\left(S_{w}\right)$. on the singular matrix $\left(S_{d c}\right)$ using the specified $\beta$ value, to produce a new singular matrix $\left(S_{m}\right)$. Embedding can be done with Eq. (11).

$$
S_{m}=S_{d c}+\left(S_{w} \cdot \beta\right)
$$

11. Perform an inverse SVD on the $\mathrm{T}(0,0)$ matrix $\left(M_{d c}\right)$ so that you get a new $\mathrm{T}(0,0)$ matrix that has been embedded with a watermark, where the singular matrix $\left(S_{d c}\right)$ is replaced with $S_{m}$.

12. Next, return the $T(0,0)$ value for each of the appropriate Tchebichef blocks, then perform the inverse Tchebichef transformation with Eq. (12) to get the watermarked frame.

$$
S_{(i, j)}=\sum_{i=0}^{7} \sum_{j=0}^{7} T_{x y} t_{x}(i) t_{y}(j)
$$

13. Convert the color space from $\mathrm{YCbCr}$ to RGB on the watermarked frame with Eq. 13.

$$
\begin{gathered}
{\left[\begin{array}{l}
R \\
G \\
B
\end{array}\right]=\left[\begin{array}{ccc}
1 & 0 & 1.402 \\
1 & -0.34414 & -0.7414 \\
1 & 1.772 & 0
\end{array}\right]} \\
{\left[\begin{array}{c}
Y \\
C b \\
C r
\end{array}\right]-\left[\begin{array}{c}
0 \\
128 \\
128
\end{array}\right]}
\end{gathered}
$$

14. Replace the original frame with a watermarked frame to get a watermarked video.

\subsection{Extraction scheme}

In the extraction scheme, several inputs are required, namely the original cover video, watermark, and LCG key. The extraction process is carried out using a non-blind method, so a watermarked video is also required in the extraction scheme. To see the workflow of the proposed embedding method, see Fig. 2. Whereas in detail the process is described as follows. 


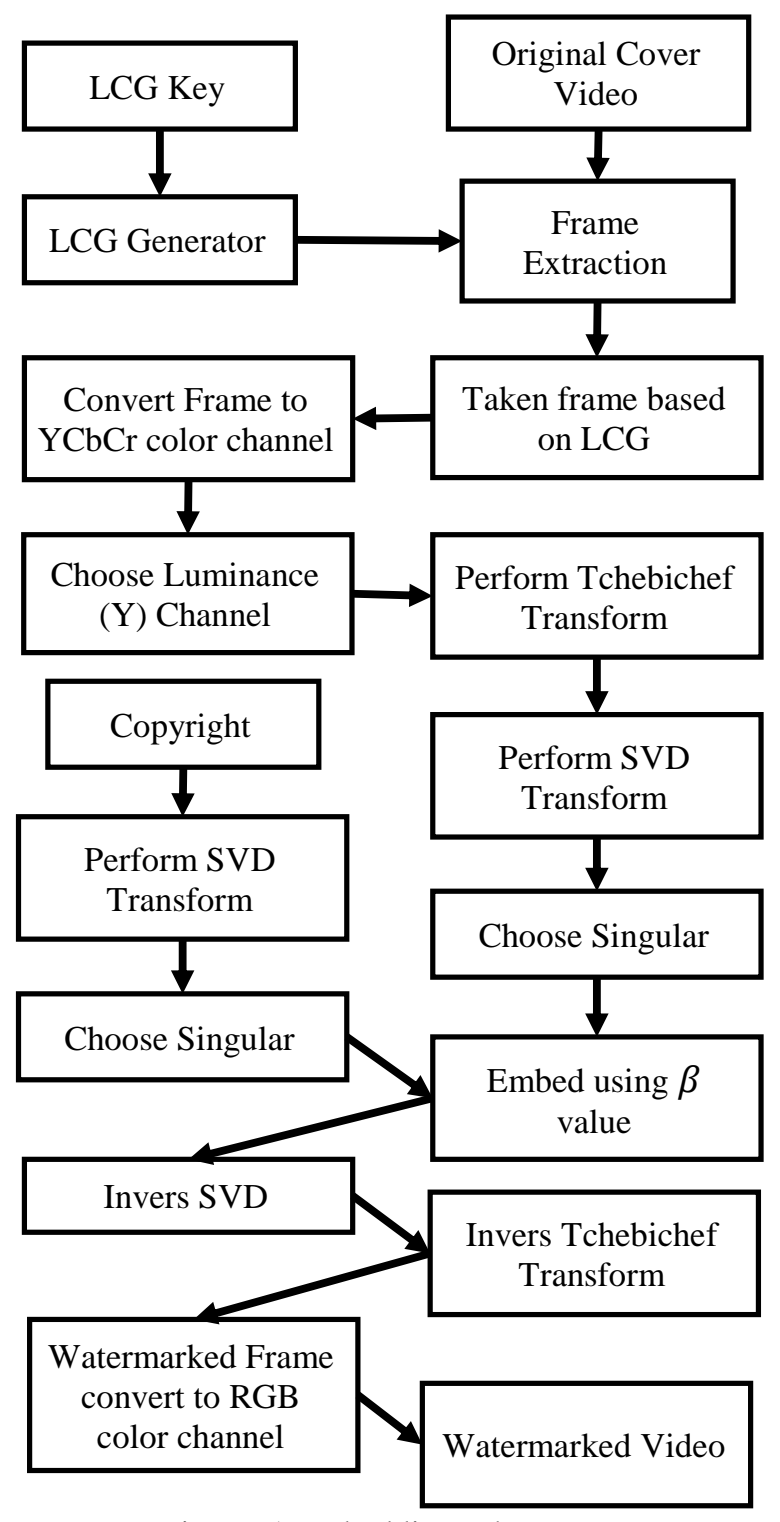

Figure. 1 Embedding scheme

1. Read the original cover video and watermarked video, get data such as the number of frames and video dimensions for the extraction process.

2. Input variables $a$ and $b$ as LCG keys, then generate pseudo-random values using Eq. (1). Get the first two values from the pseudo-random, get the average value then round it down (same as step 2 of the embedding scheme).

3. Extract both videos, then take the frame based on the value generated in step 2 .

4. Convert RGB color space to $\mathrm{YCbCr}$ on the original frame and watermarked frame.

5. Perform steps 5 to 7 on the embed scheme on the original frame and the watermarked frame. So we get the original singular matrix $\left(S_{d c}\right)$ and watermarked singular matrix $\left(S_{m}\right)$.

6. Extract both singular frame matrices to get the singular watermark matrix, do it with Eq. (14).

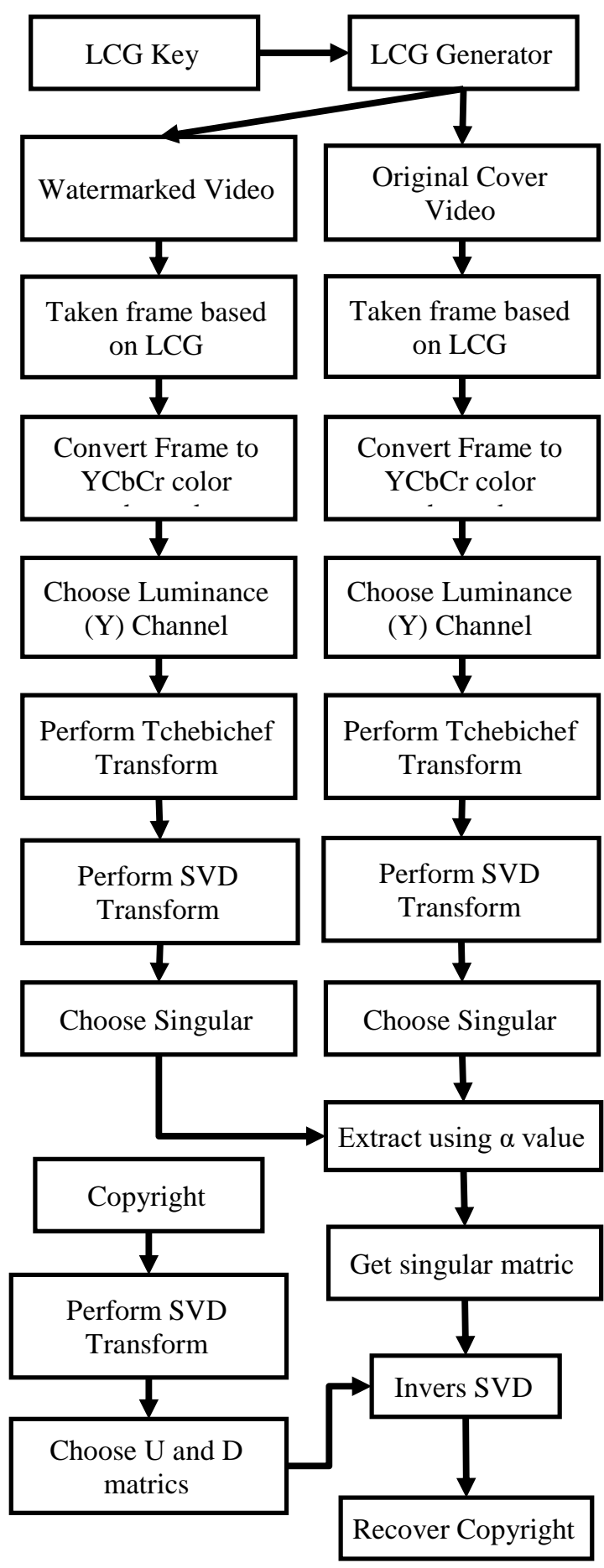

Figure. 2 Extraction scheme

$$
S_{w}=\left(S_{w}-S_{d c}\right) / \beta
$$

Where the value of $\beta$ is the same as the watermark embedding process

7. Pada sisi lain, baca watermark, lakukan transforasi SVD, untuk mendapatkan matrik $\mathrm{H}$ dan Matrik V.

8. Get recover watermark by inverse SVD based on the singular matrix $\left(S_{w}\right)$ 


\section{Implementation and analysis}

In this section, the initial step taken is data collection. Standard video data is carried out in this research so that comparisons can be made with the previously set. Standard videos compiled from the media.xiph.org/video/derf/ page. All downloaded videos have a resolution of $384 \times 288$ pixels (4: 3 ) and have a $\mathrm{y} 4 \mathrm{~m}$ extension.

In the implementation process, the Matlab $\mathrm{R} 2015 \mathrm{a}$ application is used with a laptop device that has AMD A12-9720P processor specifications and 8GB memory (7.39 GB usable). Because Matlab cannot read the $\mathrm{y} 4 \mathrm{~m}$ extension, it is necessary to convert the video to AVI with the Total Video Audio Converter 4 application, then read and rewrite it using the Matlab VideoReader and VideoWriter functions with Uncompress AVI mode. The entire video has 300 frames. Fig. 3 presents the first frame of each video used. The watermark image is a binary image with a resolution of $48 \times 36$ pixels. The watermark image is presented in Fig. 4.

After the entire dataset is ready, the proposed method is implemented. For example, for the values of the variables, $a$, and $b$ in the LCG key used are 2006336 and 2012404, so the values of $Z 1=240$ and $\mathrm{Z} 2=244$ are obtained so that the selected frame for embedding is the $242^{\text {nd }}$ frame. While the $\beta$ value used in each video is the same, which is 20. After embedding the video, the quality is measured using the peak signal to noise ratio (PSNR) and mean square error (MSE). The higher PSNR value indicates the watermarked video quality that is more similar to the original video, while the MSE value should be the opposite, where the smaller MSE value (closer to 0) indicates that the watermarked video quality is playing well. The MSE and PSNR values on the video can be calculated by Eq. (15) and Eq. (16). The results of the PSNR and MSE calculations are presented in Table 1.

$$
\begin{aligned}
& M S E= \\
& \begin{array}{c}
\frac{1}{w \times h \times c \times f} \sum_{i=1}^{w} \sum_{j=1}^{h} \sum_{k=1}^{c} \sum_{l=1}^{f} \| V_{w}(i, j, k, l)- \\
V_{o}(i, j, k, l) \|^{2}
\end{array} \\
& \operatorname{PSNR}_{(d B)}=10 \times \log 10\left(\frac{255^{2}}{\sqrt{M S E}}\right)
\end{aligned}
$$

Where $w$ is the width of the video frame, $h$ is the height of the video frame, $c$ is the number of channels (red, green, and blue) in the frame, and $f$ is the number of video frames. $V_{w}$ is watermarked video, $V_{o}$ is original cover video, while $i, j, k, l$ are the coordinates of the pixel values.

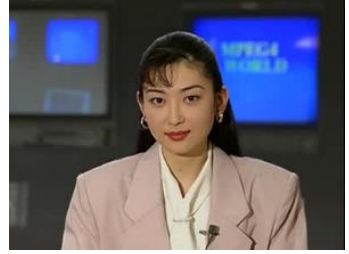

(a)

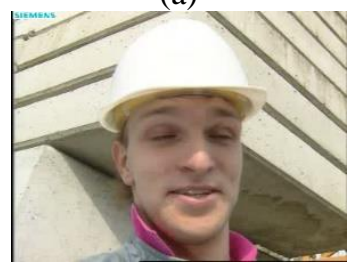

(c)

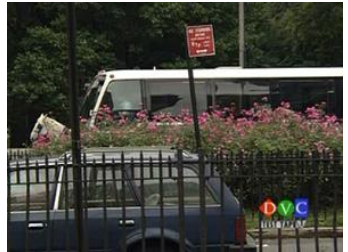

(b)

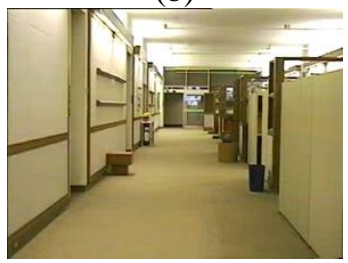

(d)
Figure. 3 Video cover used: (a) akiyo, (b) bus, (c) foreman, and (d) hall

\section{(ㄱ)}

Figure. 4 Watermark image used

Table 1. PSNR and MSE results of watermarked video

\begin{tabular}{|c|c|c|}
\hline Video & PSNR (dB) & MSE \\
\hline akiyo & 46.951007 & 1.312138 \\
\hline bus & 54.451713 & 0.233297 \\
\hline foreman & 51.107109 & 0.503929 \\
\hline hall & 51.298101 & 0.482248 \\
\hline average & 50.951983 & 0.632903 \\
\hline
\end{tabular}

Based on the results presented in Table 1, it appears that the PSNR values presented are quite varied, from $46.951007 \mathrm{~dB}$ to $54.451713 \mathrm{~dB}$. The PSNR value is not stable enough for each video, but the lowest value is still above $40 \mathrm{~dB}$, which shows that the PSNR value is still in the excellent category, as well as the MSE value which is entirely below 1 .

In the next test, the extraction process is carried out, in which two kinds of extraction, namely extraction without attack and extraction which is done after the watermarked video is manipulated. Fig. 5 shows a sample frame of one of the videos, the one with no attacks and the one with multiple attacks. All attacks are carried out with Matlab functions.

The information presented in Fig. 3 is the type of attack carried out, where the attack index sequence is also the same as that shown in Table 2 and Table 3. In Table 2, the value of the robustness quality calculation using the correlation coefficient (cc) measurement tool is presented,

where $\mathrm{cc}$ is measured by Eq. (17), whereas in Table 3 the visually extracted watermark is presented.

$$
c c=\frac{\sum_{i} \sum_{j}\left(w_{i j}-\bar{w}\right)\left(e_{i j}-\bar{e}\right)}{\sqrt{\left(\sum_{i} \sum_{j}\left(w_{i j}-\bar{w}\right)^{2}\right)\left(\sum_{i} \sum_{j}\left(e_{i j}-\bar{e}\right)^{2}\right)}}
$$




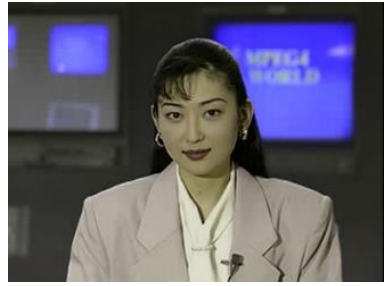

(a)

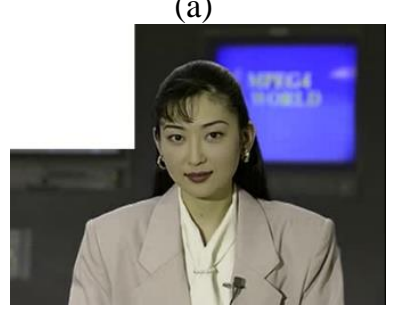

(c)

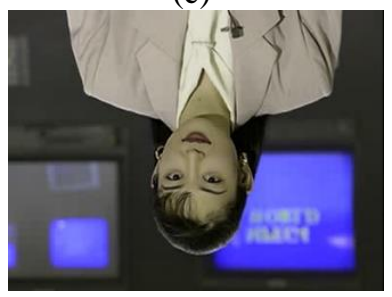

(e)

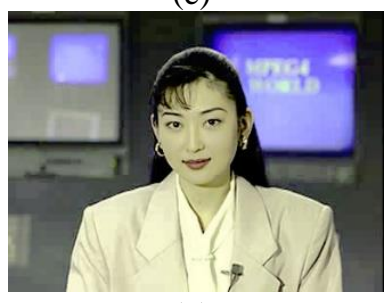

(g)

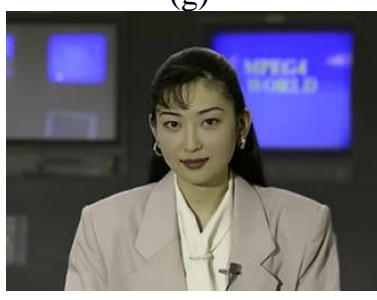

(i)

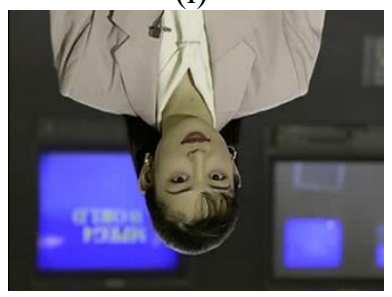

(k)

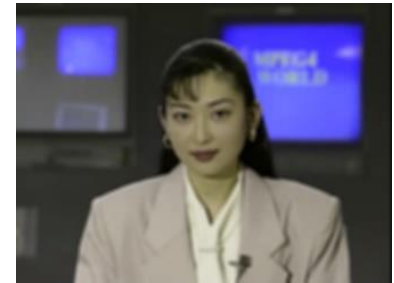

(b)

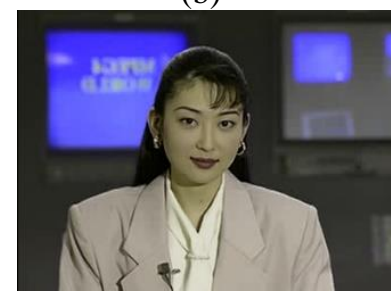

(d)

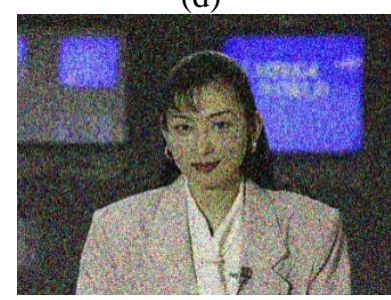

(f)

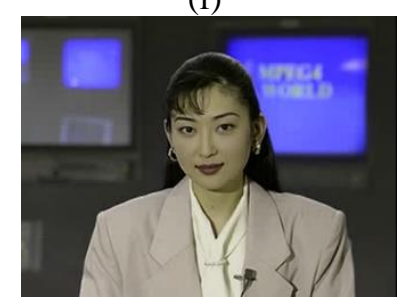

(h)

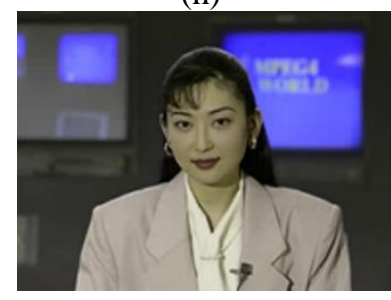

(j)

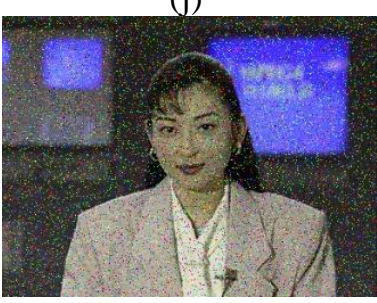

(1)
Figure. 3 Sample frame of Akiyo video after: (a) no attack, (b) blurred (disk, 3), (c) cropped $128 \times 128$ pixels,

(d) flip horizontal, (e) flip vertical, (f) gaussian noise

0.05, (g) histogram equalization, (h) motion JPEG AVI $\mathrm{Q}=10$, (i) motion JPEG AVI $\mathrm{Q}=50,(\mathrm{j})$ rescaling $50 \%,(\mathrm{k})$ rotate $180^{\circ}$, and (l) salt and pepper 0.1

Where $w$ is original watermark image, $e$ is extracted watermark image, $i, j$ is the watermark image resolution, $\bar{w}$ is mean of $w_{i j}$ and $\bar{e}$ is mean of $e_{i j}$
Table 2. CC results of extracted watermark

\begin{tabular}{|c|c|c|c|c|}
\hline 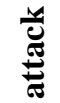 & akiyo & bus & foreman & hall \\
\hline $\mathrm{a}$ & 0.939140 & 0.974698 & 0.930655 & 0.870101 \\
\hline$b$ & 0.937504 & 0.974160 & 0.929739 & 0.867410 \\
\hline $\mathrm{c}$ & 0.917044 & 0.943560 & 0.921919 & 0.872875 \\
\hline $\mathrm{d}$ & 0.939020 & 0.974227 & 0.930569 & 0.870632 \\
\hline $\mathrm{e}$ & 0.939140 & 0.974698 & 0.930655 & 0.870101 \\
\hline $\mathrm{f}$ & 0.932648 & 0.967890 & 0.925550 & 0.862816 \\
\hline $\mathrm{g}$ & 0.939680 & 0.966852 & 0.953798 & 0.913909 \\
\hline $\mathrm{h}$ & 0.939373 & 0.974709 & 0.930709 & 0.870151 \\
\hline $\mathrm{i}$ & 0.939118 & 0.974712 & 0.930727 & 0.869952 \\
\hline $\mathrm{j}$ & 0.939026 & 0.974683 & 0.930573 & 0.869625 \\
\hline $\mathrm{k}$ & 0.938344 & 0.974227 & 0.930569 & 0.870632 \\
\hline 1 & 0.932445 & 0.968456 & 0.924927 & 0.862776 \\
\hline avg & 0.936040 & 0.970239 & 0.930866 & 0.872582 \\
\hline
\end{tabular}

Table 3. Extracted watermark results

\begin{tabular}{|c|c|c|c|c|}
\hline attack & akiyo & bus & foreman & hall \\
\hline $\mathrm{a}$ & (B) & ( $^{\mathrm{TH}}$ & (B) ${ }^{\mathrm{TH}}$ & (B)TH \\
\hline $\mathrm{b}$ & (B) ${ }^{\mathrm{T}}$ & (B) & $\left(\mathrm{B}^{\mathrm{TH}}\right.$ & (B) \\
\hline $\mathrm{c}$ & (B) & (B) $^{\mathrm{TH}}$ & (B) $^{\mathrm{TH}}$ & (B) \\
\hline $\mathrm{d}$ & (B) & (B) $^{14}$ & (B) $^{\mathrm{T}}$ & (B)TH \\
\hline $\mathrm{e}$ & (B) & $\mathrm{B}^{\mathrm{TH}}$ & (B) $^{\mathrm{TH}}$ & (E)T세 \\
\hline $\mathrm{f}$ & (B) & (B) & (B) & (B)TH \\
\hline $\mathrm{g}$ & (B) & $B^{T H}$ & $\mathrm{~B}^{\mathrm{TH}}$ & (B) \\
\hline $\mathrm{h}$ & (B) & (B) $^{\mathrm{TH}}$ & $\left(\mathrm{C}^{\mathrm{TH}}\right.$ & (B)TH \\
\hline $\mathrm{i}$ & (B) & (B) $^{\mathrm{TH}}$ & $\left(\mathrm{B}^{\mathrm{TH}}\right.$ & (B)Th \\
\hline $\mathrm{j}$ & (B) $^{\mathrm{TH}}$ & $\mathrm{B}^{\mathrm{TH}}$ & (B) $^{\mathrm{TH}}$ & (B)Th \\
\hline $\mathrm{k}$ & (B) & (B) $^{14}$ & (B) & (B)TH \\
\hline 1 & (B) & (B) $^{\mathrm{T}}$ & $\mathrm{B}^{\mathrm{TH}}$ & (B) \\
\hline
\end{tabular}

Based on the results presented in Table 2 and Table 3, it appears that none of the watermark extraction results get a perfect score. The brightness level of the color on the frame greatly affects the results of watermark embedding and extraction. In the akiyo, foreman, and hall video have colors that tend to be brighter, while the bus video has a darker 
color composition. Initial extraction and $\beta$ value in watermarked video greatly affect all watermark extraction. However, this proposed method has advantages in the aspect of robustness, where all extraction by the attack has a value close to extraction without attack. So, even though the extraction cannot be perfect, this method is proven to have strong robustness to various kinds of attacks, especially geometric attacks such as flip and rotate, this is due to the use of SVD transformations. In the attack of Gaussian noise, salt and pepper, rescaling, compression, blur, and histogram equalization this method is quite strong because the embedding is done on the luminance $(\mathrm{Y})$ channel, where the Tchebichef transformation results of 0.0 coordinates in each $8 \times 8$ matric are collected. So that the watermark is right on the core of the image. This makes watermarks more difficult to remove with various manipulation models. SVD also plays a role in increasing the imperceptibility of watermarked videos. An interesting finding is that in the extraction results of the histogram equalization attack (attack no. g), it was found that the cc value was better than the extraction without attack, this was due to the imperfect extraction results of watermarked video without attack. The use of the LCG method also adds watermark security because the watermark insertion is done randomly on a certain frame based on the key. Furthermore, at this stage, comparisons are also carried out with several previous methods, such as research conducted by Li et al. [28] and Bhardwaj et al. [29]. Comparisons are carried out on standard video covers such as akiyo, foreman, and hall, while for the type of watermark a binary image is used. It should be noted that in the comparisons presented in Table 4 and Table 5, some have blank values (marked with "-"), this is because not all test results in the dataset were presented in previous research.

Based on the results presented in Table 4 it appears that the proposed method has advantages in the imperceptibility aspect. On the method that $\mathrm{Li}$ et al. [28] the embedding was carried out at the DC coefficient or coordinates 0.0 , this research was also carried out, but the transformation was carried out with Tchebichef and combined with SVD to produce a more optimal watermarking. This research also uses frame selection techniques such as that done by Bhardwaj et al. [29], the aim is to improve the quality of imperceptibility, where it is proven that the PSNR value increases significantly.

From the results presented in Table 5, the robustness aspect of the proposed method is strong against Gaussian noise attacks, cropping, and histogram equalization. Rescaling and salt and pepper attacks on the proposed method are actually
Table 4. PSNR (dB) results of watermarked video

\begin{tabular}{|c|c|c|c|}
\hline \multirow{2}{*}{ Video } & \multicolumn{3}{|c|}{ Method } \\
\cline { 2 - 4 } & $\begin{array}{c}\text { Li et al. } \\
{[28]}\end{array}$ & $\begin{array}{c}\text { Bhardwaj } \\
\text { et al [29] }\end{array}$ & Proposed \\
\hline akiyo & - & 41.50 & 46.9510 \\
\hline foreman & 37.3423 & 41.51 & 51.1071 \\
\hline hall & - & 41.72 & 51.2981 \\
\hline
\end{tabular}

Table 5. Correlation results of extracted watermark from foreman video

\begin{tabular}{|c|c|c|c|}
\hline \multirow{2}{*}{ Attack } & \multicolumn{3}{|c|}{ Method } \\
\cline { 2 - 4 } & $\begin{array}{c}\text { Yassin et } \\
\text { al. [32] }\end{array}$ & $\begin{array}{c}\text { Bhardwaj } \\
\text { et al [29] }\end{array}$ & Proposed \\
\hline No attack & 0.994 & $\mathbf{1 . 0 0 0}$ & 0.931 \\
\hline $\begin{array}{c}\text { Gaussian } \\
\text { Noise }\end{array}$ & 0.562 & 0.810 & $\mathbf{0 . 9 2 6}$ \\
\hline Crop & 0.785 & 0.900 & $\mathbf{0 . 9 2 2}$ \\
\hline Rescalling & 0.756 & $\mathbf{0 . 9 9 0}$ & 0.931 \\
\hline $\begin{array}{c}\text { Salt and } \\
\text { pepper }\end{array}$ & - & $\mathbf{0 . 9 3 9}$ & 0.925 \\
\hline $\begin{array}{c}\text { Histogram } \\
\text { Equalization }\end{array}$ & - & 0.8867 & $\mathbf{0 . 9 5 4}$ \\
\hline
\end{tabular}

very strong, but because the extraction results of the no attack cannot be done perfectly, it makes the results of the salt and pepper and rescaling attack values look smaller.

\section{Conclusions}

From the results of the test that have been conducted, the Tchebichef transform and SVD schemes based on LCG generator are proven to produce robust, imperceptible, and secure watermarking. With the frame selection technique as has been done in previous research, this method can increase the imperceptibility value of the PSNR value to more than $50 \mathrm{~dB}$. The level of robustness is also excellent proved by the difference cc value close to the value of no attack. However, this scheme still needs to be improved to get better watermark extraction results. The value of $\beta$ has an important role in the extraction and embedding results so that in further research it is necessary to use a system to provide the most optimal automatic value so that it can produce even better results, both in the embedding scheme and the extraction scheme.

\section{Conflicts of Interest}

We wish to confirm that there are no known conflicts of interest associated with this publication and there has been no significant financial support for this work that could have influenced its outcome. We confirm also that the manuscript has been read and 
approved by all named authors and that there are no other persons who satisfied the criteria for authorship but are not listed.

\section{Acknowledgments}

The authors would like to express very special thanks to the Ministry of Research, Technology, and the Higher Education Republic of Indonesia for providing financial support of this research project with letter number: 043/A38.04/UDN-09/IV/2020

\section{References}

[1] B. Latha, V. Reddy Dasari, and D. Avula, "Robust Video Watermarking Using Secret Sharing, SVD, DWT, and Chaotic Firefly Algorithm", International Journal of Intelligent Engineering and Systems, Vol. 11, No. 1, pp. 171-180, 2018.

[2] I. Assini, A. Badri, K. Safi, A. Sahel, and A. Baghdad, "A Robust Hybrid Watermarking Technique for Securing Medical Image", International Journal of Intelligent Engineering and Systems, Vol. 11, No. 3, pp. 169-176, 2018.

[3] N. N. Mood and V. Subbareddy Konkula, "A Novel Image Watermarking Scheme Based on Wavelet Transform and Genetic Algorithm", International Journal of Intelligent Engineering and Systems, Vol. 11, No. 3, pp. 251-260, 2018.

[4] T. Venugopal, V. Siva, and K. Reddy, "Image Watermarking Using Two Level Encryption Method Based on Chaotic Logistic Mapping and Rivest Shamir Adleman Algorithm”, International Journal of Intelligent Engineering and Systems, Vol. 11, No. 6, pp. 271-281, 2018.

[5] M. Asikuzzaman and M. R. Pickering, "An Overview of Digital Video Watermarking", IEEE Transactions on Circuits and Systems for Video Technology, Vol. 28, No. 9. Institute of Electrical and Electronics Engineers Inc., pp. 2131-2153, 2018.

[6] D. R. I. M. Setiadi, "Improved payload capacity in LSB Image Steganography uses dilated hybrid edge detection", J. King Saud Univ. Comput. Inf. Sci., 2019.

[7] A. Susanto, D. R. I. M. Setiadi, C. A. Sari, and E. H. Rachmawanto, "Hybrid method using HWT-DCT for image watermarking", In: Proc. of 2017 5th International Conf. on Cyber and IT Service Management, CITSM 2017, 2017.

[8] L. Agilandeeswari and K. Ganesan, "A robust color video watermarking scheme based on hybrid embedding techniques", Multimed. Tools Appl., Vol. 75, No. 14, pp. 8745-8780, 2016.
[9] K. Fares, K. Amine, and E. Salah, "A robust blind color image watermarking based on Fourier transform domain”, Optik (Stuttg)., Vol. 208, p. 164562, 2020.

[10] X. Y. Wang, C. P. Wang, H. Y. Yang, and P. P. $\mathrm{Niu}$, "A robust blind color image watermarking in quaternion Fourier transform domain", J. Syst. Softw., Vol. 86, No. 2, pp. 255-277, 2013.

[11] X. Zhang, Q. Su, Z. Yuan, and D. Liu, "An efficient blind color image watermarking algorithm in spatial domain combining discrete Fourier transform", Optik (Stuttg)., p. 165272, Jul. 2020.

[12] Z. Yuan, D. Liu, X. Zhang, and Q. Su, "New image blind watermarking method based on two-dimensional discrete cosine transform", Optik (Stuttg)., Vol. 204, p. 164152, 2020.

[13] D. Ariatmanto and F. Ernawan, "Adaptive scaling factors based on the impact of selected DCT coefficients for image watermarking", $J$. King Saud Univ. - Comput. Inf. Sci., 2020.

[14] A. Setyono and D. R. I. M. Setiadi, "Image Watermarking using Discrete WaveletTchebichef Transform", Indones. J. Electr. Eng. Comput. Sci., Vol. 16, No. 3, pp. 1416-1423, Dec. 2019.

[15] A. Setyono and D. R. I. M. Setiadi, "Tchebichef Image Watermarking based on 2-level Haar Wavelet", In: Proc. of the 4th International Conf. on Contemporary Computing and Informatics, IC3I 2019, pp. 16-21, 2019.

[16] H. T. Hu, L. Y. Hsu, and H. H. Chou, "An improved SVD-based blind color image watermarking algorithm with mixed modulation incorporated”, Inf. Sci. (Ny)., vol. 519, pp. 161182, May 2020.

[17] A. Setyono and D. R. I. M. Setiadi, “An Image Watermarking Method Using Discrete Tchebichef Transform and Singular Value Decomposition Based on Chaos Embedding", International Journal of Intelligent Engineering and Systems, Vol. 13, No. 2, pp. 140-150, 2020.

[18] B. Xiao, J. Luo, X. Bi, W. Li, and B. Chen, "Fractional discrete Tchebyshev moments and their applications in image encryption and watermarking", Inf. Sci. (Ny)., Vol. 516, pp. 545-559, 2020.

[19] V. Adul and E. Mwangi, "A robust video watermarking approach based on a hybrid SVD/DWT technique", in 2017 IEEE AFRICON: Science, Technology and Innovation for Africa, AFRICON 2017, pp. 309-313, 2017.

[20] S. Jiao, C. Zhou, Y. Shi, W. Zou, and X. Li, "Review on optical image hiding and 
watermarking techniques", Opt. Laser Technol., Vol. 109, pp. 370-380, 2019.

[21] N. M. Makbol and B. E. Khoo, "A new robust and secure digital image watermarking scheme based on the integer wavelet transform and singular value decomposition", Digit. Signal Process., Vol. 33, pp. 134-147, 2014.

[22] O. Jane, E. Elbaşi, and H. G. İlk, "Hybrid NonBlind Watermarking Based on DWT and SVD", J. Appl. Res. Technol., Vol. 12, No. 4, pp. 750761, 2014.

[23] C. A. Sari, E. H. Rachmawanto, and D. R. I. M. Setiadi, "Robust and imperceptible image watermarking by DC coefficients using singular value decomposition", In: Proc. of International Conf. on Electrical Engineering, Computer Science and Informatics (EECSI), 2017.

[24] D. R. I. M. Setiadi, T. Sutojo, E. H. Rachmawanto, and C. A. Sari, "Fast and efficient image watermarking algorithm using discrete tchebichef transform", In: Proc. of 2017 5th International Conf. on Cyber and IT Service Management (CITSM), pp. 1-5, 2017.

[25] R. O. Preda and D. N. Vizireanu, "A robust digital watermarking scheme for video copyright protection in the wavelet domain", Meas. J. Int. Meas. Confed., Vol. 43, No. 10, pp. 1720-1726, 2010.

[26] P. A. M. Oliveira, R. J. Cintra, F. M. Bayer, S. Kulasekera, and A. Madanayake, "A Discrete Tchebichef Transform Approximation for Image and Video Coding", IEEE Signal Process. Lett., Vol. 22, No. 8, pp. 1137-1141, 2015.

[27] P. A. M. Oliveira, R. J. Cintra, F. M. Bayer, S. Kulasekera, and A. Madanayake, "LowComplexity Image and Video Coding Based on an Approximate Discrete Tchebichef Transform", IEEE Trans. Circuits Syst. Video Technol., Vol. 27, No. 5, pp. 1066-1076, 2017.

[28] J. Li, W. Yongbin, and D. Shusen, "Video watermarking algorithm based DC coefficient", In: Proc. of 2017 2nd International Conf. on Image, Vision and Computing, ICIVC 2017, pp. 454-458, 2017.

[29] A. Bhardwaj, V. S. Verma, and R. K. Jha, "Robust video watermarking using significant frame selection based on coefficient difference of lifting wavelet transform", Multimed. Tools Appl., Vol. 77, No. 15, pp. 19659-19678, 2018.

[30] E. H. Rachmawanto, K. Prasetyo, C. A. Sari, D. R. I. M. Setiadi, and N. Rijati, "Secured PVD video steganography method based on AES and linear congruential generator", in International Seminar on Research of Information Technology and Intelligent Systems, pp. 163-167, 2018.
[31] C. A. Sari, U. Utariyanto, D. R. I. M. Setiadi, E. H. Rachmawanto, and M. K. Sarker, "Robust IHWT-CRT Image Watermarking using $\mathrm{YCbCr}$ Color Space", In: Proc. of J. Phys. Conf. Ser., Vol. 1501, 2020.

[32] N. I. Yassin, N. M. Salem, and M. I. El Adawy, "QIM blind video watermarking scheme based on Wavelet transform and principal component analysis", Alexandria Eng. J., Vol. 53, No. 4, pp. 833-842, 2014. 